\title{
MicroRNA-148a/b-3p regulates angiogenesis by targeting neuropilin-1 in endothelial cells
}

\author{
Hyejeong Kim (1)', Yeongrim Ko ${ }^{1}$, Hyojin Park', Haiying Zhang ${ }^{1}$, Yoonjeong Jeong ${ }^{1}$, Yeomyeong Kim', Minyoung Noh',
} Songyi Park', Young-Myeong Kim² and Young-Guen Kwon'

\begin{abstract}
MicroRNAs (miRs) are crucial regulators of vascular endothelial cell (EC) functions, including migration, proliferation, and survival. However, the role of most miRs in ECs remains unknown. Using RNA sequencing analysis, we found that miR-148a/b-3p expression was significantly downregulated during the differentiation of umbilical cord blood mononuclear cells into outgrowing ECs and that decreased miR-148a/b-3p levels were closely related to EC behavior. Overexpression of miR-148a/b-3p in ECs significantly reduced migration, filamentous actin remodeling, and angiogenic sprouting. Intriguingly, the effects of decreased miR-148a/b-3p levels were augmented by treatment with vascular endothelial growth factor (VEGF). Importantly, we found that miR-148a/b-3p directly regulated neuropilin-1 (NRP1) expression by binding to its $3^{\prime}$-untranslated region. In addition, because NRP1 is the coreceptor for VEGF receptor 2 (VEGFR2), overexpression of miR-148a/b-3p inhibited VEGF-induced activation of VEGFR2 and inhibited its downstream pathways, as indicated by changes to phosphorylated focal adhesion kinase (FAK), extracellular signalregulated kinase (ERK), and p38 mitogen-activated protein kinase. Collectively, our results demonstrate that miR-148a/ $\mathrm{b}-3 \mathrm{p}$ is a direct transcriptional regulator of NRP1 that mediates antiangiogenic pathways. These data suggest that miR148a/b-3p is a therapeutic candidate for overcoming EC dysfunction and angiogenic disorders, including ischemia, retinopathy, and tumor vascularization.
\end{abstract}

\section{Introduction}

Angiogenesis is the process by which new blood vessels form from the existing vasculature in response to angiogenic stimuli. Angiogenesis is crucial for ensuring healthy physiological processes, including embryo development and tissue repair, but it also has pathological roles in promoting tumor growth ${ }^{1}$. Vascular endothelial growth factor (VEGF), a very specific mitogen for vascular endothelial cells (ECs), stimulates angiogenesis by binding to VEGF receptor 1 (VEGFR1) and VEGF receptor 2 (VEGFR2) and their coreceptors, neuropilin-1 (NRP1) and neuropilin-2 (NRP2) ${ }^{2}$. In particular, VEGF/VEGFR2induced signal transduction is a major ligand-receptor complex in the VEGF system. Activated VEGF/

\footnotetext{
Correspondence: Young-Guen Kwon (ygkwon@yonsei.ac.kr)

'Department of Biochemistry, College of Life Science and Biotechnology,

Yonsei University, Seoul 03722, Korea

${ }^{2}$ Vascular System Research Center, Kangwon National University, Chuncheon,

Kangwon 24341, Republic of Korea
}

VEGFR2 signaling in ECs promotes proliferation, migration, cytoskeletal reorganization, differentiation, and the formation of new vasculature. Mounting evidence suggests that NRP1, which is a known coreceptor of VEGF, also plays an important role in angiogenesis. NRP1 can enhance VEGF-mediated activity and downstream signaling of VEGFR2 in ECs. Furthermore, the PDZ-binding domain in the cytoplasmic region of NRP1 is essential for both the formation of a stable NRP1/VEGFR2 complex $^{3,4}$ and the trafficking of endocytosed VEGFR2 ${ }^{5}$. Overexpression of NRP1 in tumor cells has also been shown to enhance tumor angiogenesis ${ }^{6,7}$.

MicroRNAs (miRs) are single-stranded, noncoding RNA molecules that have crucial roles in regulating gene expression; therefore, miRs control diverse cellular and metabolic pathways ${ }^{8}$. Recently, many studies have focused on identifying the relationship between a specific miR and the biological function of its target mRNA. Dysregulation of miRs leads to pathological processes, and some miRs 
are closely related to the onset of cancer because they modulate the expression of tumor suppressors. For example, miR-148a-3p is a tumor suppressor that is significantly downregulated in several types of cancers, including hepatocellular carcinoma, gastric cancer, and ovarian cancer ${ }^{9-11}$. miR-148b-3p is also reported to participate in the regulation of tumorigenesis. Indeed, miR$148 \mathrm{~b}-3 \mathrm{p}$ represses tumor growth by regulating the protooncogene receptor tyrosine kinase, KIT, in gastrointestinal stromal tumors ${ }^{12}$. Although there has been extensive research on miR-148a/b in the context of cancer, its angiogenic properties remain largely unknown.

In the present study, we report the expression of miR148a/b-3p during the differentiation of umbilical cord blood mononuclear cells (UCB-MNCs) into outgrowing ECs (OECs) and investigate the effects of miR-148a/b-3p on EC migration, F-actin remodeling, and angiogenic sprouting. We also identified target genes regulated by $\mathrm{miR}-148 \mathrm{a} / \mathrm{b}-3 \mathrm{p}$. Finally, we evaluated the role of miR$148 \mathrm{a} / \mathrm{b}-3 \mathrm{p}$ in the VEGF-induced VEGFR2 signaling pathway. Our findings indicate that miR-148a/b-3p may be an interesting therapeutic target, particularly in vascular disease that is characterized by upregulated or aberrant angiogenesis.

\section{Materials and methods}

Isolation and culture of UCB-MNCs, OECs, and human umbilical vein ECs (HUVECs)

Approximately, $50 \mathrm{~mL}$ of human umbilical cord blood (HUCB) was collected by gravitational flow from umbilical cords that were still attached to the placenta. To separate UCB-MNCs from HUCB, the Ficoll-Paque density gradient method was used ${ }^{13,14}$. The isolated UCBMNCs were resuspended in endothelial basal medium-2 (EBM-2, Clonetics Cell Systems). The complete media contained $20 \%$ heat-inactivated fetal bovine serum (FBS, HyClone), human VEGF, human fibroblast growth factor$B$, human epidermal growth factor (EGFR), insulin-like growth factor 1 (IGF1), and ascorbic acid. Cells were seeded on human fibronectin (Sigma-Aldrich)-coated 6well plates at a cell density of $2 \times 10^{7}$ cells/well. After 3 days, nonadherent cells were washed away, and the media was changed every 2 days thereafter. After OEC colonies were established, samples were prepared for RNA-seq.

HUVECs were purchased from Lonza (C2517A; Walkersville, MD) and cultured in EGM-2 media (CC-3156, Lonza). The media was supplemented with the EGM-2 SingleQuot kit (CC-4176, Lonza), with 5\% FBS, and 1\% penicillin/streptomycin. Cells were routinely passaged at 80-90\% confluency, and cells between passages 3 and 7 were used for experiments. Cells were maintained at $37^{\circ} \mathrm{C}$ in a humidified atmosphere containing $5 \% \mathrm{CO}_{2}$.

\section{HUVEC transfection with mimic-microRNAs}

HUVECs (70\% confluent) were transfected with miRIDIAN human hsa-miR-148a-3p or miRIDIAN human hsa-miR-148b-3p (Dharmacon) by incubating with Lipofectamine (Invitrogen) for $2 \mathrm{~h}$ and $30 \mathrm{~min}$. As a control, the miRIDIAN microRNA Mimic Negative Control \#1 was transfected into HUVECs at a concentration of $80 \mu \mathrm{M}$. Cells were transfected $48 \mathrm{~h}$ prior to the assay. The MiRIDIAN human hsa-miR-148a-3p-mimic microRNA sequence was: 5'-UCAGUGCACUACAGAACUUUGU$3^{\prime}$ and the MiRIDIAN human hsa-miR-148b-3p-mimic microRNA sequence was 5'-UCAGUGCAUCACAGAAC UUUGU-3'. The MiRIDIAN microRNA Mimic Negative Control \#1 sequence was 5'-UCACAACCUCCUAGA AAGAGUAGA-3'.

\section{MicroRNA (miR) identification by RNA sequencing analysis}

Total RNA integrity was tested using an Agilent Technologies 2100 Bioanalyzer, and only RNA with an integrity number (RIN) value greater than 8 was used in further experiments. Small RNA (sRNA) sequencing libraries were prepared according to the manufacturer's instructions (Illumina Small RNA Prep kit). Samples were sequenced with an HISEQ 2000 sequencing system (Illumina). The RNA sequencing data were used to select miRs that were expressed in both humans and mice (106 miRs). We then selected miRs that had not been previously studied in ECs (35 miRs) for further evaluation.

\section{Target prediction with bioinformatics}

Computational predictions of miR-148a-3p and miR148b-3p target genes were performed using the following published algorithms: DIANA-microT-CDS (http://www. microrna.gr/microT-CDS), TargetScan (http://www. targetscan.org), and miRDB (http://www.mirdb.org).

\section{RNA isolation, reverse transcription polymerase chain reaction (RT-PCR), and semiquantitative RT-PCR}

Total miR was isolated using a miRNeasy Mini Kit (QIAGEN). RNA was isolated using Trizol (iNtRON), and RT-PCR of miRs was performed using $2 \times$ Maxima SYBR Green/ROX qPCR Master Mix (Thermo Scientific, K0221). All results were normalized to GAPDH expression levels. The PCR primers are listed in Table 1.

\section{Cell migration assay}

To evaluate EC motility, migration assays were performed. For the Transwell migration assay, HUVECs transfected with either the miR-148a/b mimics or the miR-control were starved in M199 media (HyClone) that contained 1\% FBS for $6 \mathrm{~h}$. The HUVECs were seeded at $1 \times 10^{5}$ cells/well in the upper chamber of a 24-well Transwell plate that was coated with $0.1 \%$ gelatin. The 
Table 1 Sequence of RT-PCR primers

\begin{tabular}{|c|c|c|c|}
\hline \multirow[t]{6}{*}{ hsa-miR-148a } & \multirow[t]{3}{*}{$3 p$} & Stem-loop & 5'-GTCGTATCCAGTGCAGGGTCCGAGGTATTCGCACTGGATACGACACAAAG-3' \\
\hline & & FW & 5'-CGGCGGTCAGTGCACTACAGA-3' \\
\hline & & REV & 5'-GTGCAGGGTCCGAGGT-3' \\
\hline & \multirow[t]{3}{*}{$5 p$} & Stem-loop & 5'-GTCGTATCCAGTGCAGGGTCCGAGGTATTCGCACTGGATACGACAGTCGG-3' \\
\hline & & FW & 5'-CGGCGGAAAGTTCTGAGACAC-3' \\
\hline & & REV & 5'-GTGCAGGGTCCGAGGT-3' \\
\hline \multirow[t]{6}{*}{ hsa-miR-148b } & \multirow[t]{3}{*}{$3 p$} & Stem-loop & 5'-GTCGTATCCAGTGCAGGGTCCGAGGTATTCGCACTGGATACGACACAAAG-3' \\
\hline & & FW & 5'-CGGCGGTCAGTGCATCACAGA-3' \\
\hline & & REV & 5'-GTGCAGGGTCCGAGGT-3' \\
\hline & \multirow[t]{3}{*}{$5 P$} & Stem-loop & 5'-GTCGTATCCAGTGCAGGGTCCGAGGTATTCGCACTGGATACGACGCCTGA-3' \\
\hline & & FW & 5'-GCGCGCGAAGTTCTGTTATAC-3' \\
\hline & & REV & 5'-GTGCAGGGTCCGAGGT-3' \\
\hline \multirow[t]{3}{*}{ hsa-RNU6 } & & Stem-loop & 5'-GTCGTATCCAGTGCAGGGTCCGAGGTATTCGCACTGGATACGACACGATT-3' \\
\hline & & FW & $5^{\prime}-C C T G C G C A A G G A T G A C-3^{\prime}$ \\
\hline & & REV & 5'-GTGCAGGGTCCGAGGT-3' \\
\hline \multirow[t]{2}{*}{ hNRP1 } & & FW & 5'-CCCCAAACCACTGATAACTCG-3' \\
\hline & & REV & 5'-AGACACCATACCCAACATTCC-3' \\
\hline \multirow[t]{2}{*}{ hPECAM1 } & & FW & 5'-TCAGAAGGACAAGGCGATTG-3' \\
\hline & & REV & 5'-GTTATGTTGACCACGATGCTG-3' \\
\hline \multirow[t]{2}{*}{ hVEGFR2 } & & FW & 5'-CCAGTCAGAGACCCACGTIT-3' \\
\hline & & REV & 5'-TCCAGAATCCTCTTCCATGC-3' \\
\hline \multirow[t]{2}{*}{ hGAPDH } & & FW & 5'-CCACCCATGGCAAATTCC-3' \\
\hline & & REV & 5'-TCGCTCCTGGAAGATGGTG-3' \\
\hline
\end{tabular}

lower chamber contained $600 \mu \mathrm{L}$ of M199 medium supplemented with $1 \%$ FBS and 40 ng of VEGF, which served as a chemoattractant. After $4 \mathrm{~h}$ of incubation, cells that did not migrate were removed with a cotton swab. The motile cells were fixed with methanol and stained with hematoxylin and eosin. The motile cells were then counted in each of 8 microscopic regions of interest under 20x magnification using an optical microscope.

\section{Western blot analysis}

HUVECs were washed with cold $1 \times$ phosphate-buffered saline and lysed with RIPA buffer (100 mM Tris-Cl, $5 \mathrm{mM}$ EDTA, $50 \mathrm{mM} \mathrm{NaCl}, 50 \mathrm{mM} \quad \beta$-glycero-phosphate, $50 \mathrm{mM} \mathrm{NaF}, 0.1 \mathrm{mM} \mathrm{Na}_{3} \mathrm{VO}_{4}, 0.5 \% \mathrm{NP}-40,1 \%$ Triton X100 , and $0.5 \%$ sodium deoxycholate) at $4{ }^{\circ} \mathrm{C}$. Sample protein concentration was quantified using a SMART BCA Protein Assay kit (iNtRON). Next, $25 \mu \mathrm{g}$ of cell lysates was separated by sodium dodecyl sulfatepolyacrylamide gel electrophoresis and then transferred to nitrocellulose membranes. The membranes were blocked with $3 \%$ bovine serum albumin in $0.1 \%$ TBST, followed by probing with primary antibodies. The membranes were then incubated with horseradish peroxidaseconjugated goat anti-rabbit IgG or goat anti-mouse IgG (Life Science) secondary antibodies. $\beta$-actin was used as a loading control. The following primary antibodies were obtained from Cell Signaling Technology and were used at a 1:1000 dilution: NRP1 (Cat. No. 3725), phosphoVEGFR2 (Cat. No. 2478), VEGFR2 (Cat. No. 3479), phospho-FAK (Cat. No. 2541), FAK (Cat. No. 2542), phospho-p38 (Cat No. 9211), p38 (Cat No. 9212), phospho-ERK (Cat. No. 9106), and ERK (Cat. No. 9102). The other primary antibodies used were ROBO1 (1:1000, R\&D Systems, Cat No. MAB7118), ITGa5 (1:1000, Santa Cruz Biotechnology, Cat No. sc-10729), and $\beta$-actin (1:2000, Thermo Fisher Scientific; Catalog No. MA515739).

\section{F-actin visualization}

HUVECs that were transfected with either the miR$148 \mathrm{a} / \mathrm{b}$ mimics or the miR-control were starved in M199 media that contained $1 \%$ FBS for $4 \mathrm{~h}$ at $37^{\circ} \mathrm{C}$. The cells 
were then replated in 35-mm dishes coated with $10 \mu \mathrm{g} / \mathrm{mL}$ fibronectin (Sigma) and were allowed to equilibrate for $4 \mathrm{~h}$. Cells were then stimulated with $20 \mathrm{ng} / \mathrm{mL}$ VEGF (Koma Biotech) for $15 \mathrm{~min}$. The HUVECs were then fixed and incubated with rhodamine-conjugated phalloidin-594 (1:250, Invitrogen) and 4',6-diamidino-2phenylindole (DAPI; 1:1000, Duolink). Fluorescence images were captured using a Carl Zeiss confocal microscope (LSM700).

\section{Luciferase miRNA target reporter assay}

The NRP1 (NM_003873) 3' untranslated region (UTR) fragment (4938-5806, 869 base pairs) was cloned using human genomic DNA, and it was inserted into the XbaI restriction site of the pGL3-vector at the 3'UTR location (Promega). The amplification primers were as follows:

5'-GCTCTAGAGAATGCTTCTAGAAACTTCCAG C-3'

and 5'-GCTCTAGATACAGTTCAGTTCTATGTGGT TTTTATG-3'.

Luciferase constructs with mutated seed sequences were synthesized using a DNA amplification service from Bioneer. Twenty-four hours after transfection with either the miR-148a/b mimic or the miR-control, the HUVECs were cotransfected with the luciferase constructs and pRL-CMV (a luciferase control reporter vector). The transfected cells were lysed with passive lysis buffer, and luciferase activity was determined using a dual luciferase assay system (Promega).

\section{Fibrin gel bead assay}

A previously established fibrin gel bead assay ${ }^{15}$ was optimized to study angiogenesis. Briefly, 2500 Cytodex beads (GE healthcare) were incubated with $1 \times 10^{6}$ HUVECs for $4 \mathrm{~h}$ at $37^{\circ} \mathrm{C}$ and $5 \% \mathrm{CO}_{2}$. The tube was tapped every $20 \mathrm{~min}$ for $4 \mathrm{~h}$. The coated beads were then transferred to a T25 flask containing $5 \mathrm{~mL}$ of EGM-2 media, where they equilibrated overnight. The following day, the bead-coated cells were resuspended at a concentration of 200 beads $/ \mathrm{mL}$ in a $2-\mathrm{mg} / \mathrm{mL}$ fibrinogen (Sigma) solution containing $0.15 \mathrm{U} / \mathrm{mL}$ aprotinin (Sigma) and 0 or $30 \mathrm{ng} / \mathrm{mL}$ of hVEGF. Thrombin that was at a concentration of $0.625 \mathrm{U} / \mathrm{mL}$, and it was added to each well of a 24-well plate, followed by the addition of the fibrinogen/bead solution. The plate was incubated at room temperature for $5 \mathrm{~min}$. Then, it was placed in an incubator at $37^{\circ} \mathrm{C}$ and $5 \% \mathrm{CO}_{2}$ for $15 \mathrm{~min}$ to generate a clot. During the incubation, human skin fibroblasts were trypsinized and seeded on top of the fibrin gel at a concentration of 20,000 cells/well in $1 \mathrm{~mL}$ of EGM-2 supplemented with $2 \%$ FBS. The growth media was changed every other day. Sprouting was observed after 2-3 days.

\section{Cell proliferation assay}

Cell proliferation was determined by MTT assay. Briefly, HUVECs were seeded into a gelatin-coated 24well plate at $3.2 \times 10^{4}$ cells/well and incubated at $37^{\circ} \mathrm{C}$ in $5 \%$ EC growth medium (EGM-2, Lonza) overnight. After attachment, the cells were cultured with M199 (HyClone) supplemented with $1 \%$ FBS. Next, the cells were stimulated with VEGF (Koma Biotech, $20 \mathrm{ng} / \mathrm{mL}$ ) and EGF (BioLegend, $20 \mathrm{ng} / \mathrm{mL}$ ) for $24 \mathrm{~h}$. The cells were then incubated for $4 \mathrm{~h}$ at $37^{\circ} \mathrm{C}$ with MTT solution $(0.1 \mathrm{mg} / \mathrm{mL}$, Sigma) for evaluation of cell proliferation. After the 4-h incubation period, the MTT solution was removed, and a $50 \%$ dimethyl sulfoxide/ethanol solution (Sigma-Aldrich) was added $(200 \mu \mathrm{l} /$ well $)$ to solubilize formazan crystals. The absorbance was then detected at a wavelength of $540 \mathrm{~nm}$, and cell proliferation was calculated as a percentage of the control.

\section{Statistical analysis}

GraphPad Prism (version 5.1; GraphPad Software, La Jolla, CA) was used for statistical analyses. Statistical significance was determined using the unpaired Student's $t$ test, and $P$ values less than 0.05 were considered statistically significant. All experiments were performed at least three times, and representative results are shown. All data are presented as the mean \pm standard error of the mean.

\section{Results}

The expression levels of miR-148a/b-3p are reduced during UCB-MNC to OEC differentiation

To identify novel miRs that regulate gene expression in ECs, we conducted microRNA-sequencing (RNA-seq) analysis during UCB-MNC differentiation into OECs. The top 19 differentially expressed miRs were dramatically decreased or increased in OECs compared to their levels in UCB-MNCs (Fig. 1a). Among these, we focused on miR-148a/b-3p because it was significantly downregulated in OECs but had not been studied in ECs (Fig. 1b). We confirmed miR-148a/b-3p expression in UCB-MNCs and OECs using RT-PCR. Interestingly, miR-148a/b-5p was not detected in either UCB-MNCs or OECs. miR-148a/b$3 p$ was significantly downregulated in OECs compared with UCB-MNCs (Fig. 1c). These results indicate that miR-148a/b-3p is downregulated during UCB-MNC differentiation and suggest that miR-148a/b-3p may regulate EC function.

\section{NRP1, ITGa5, and ROBO1 are identified as target genes of miR-148a/b-3p}

We wanted to identify the genes that are regulated by miR148a/b-3p. To identify putative mRNA targets of miR-148a/b-3p, we used three bioinformatic algorithms: 


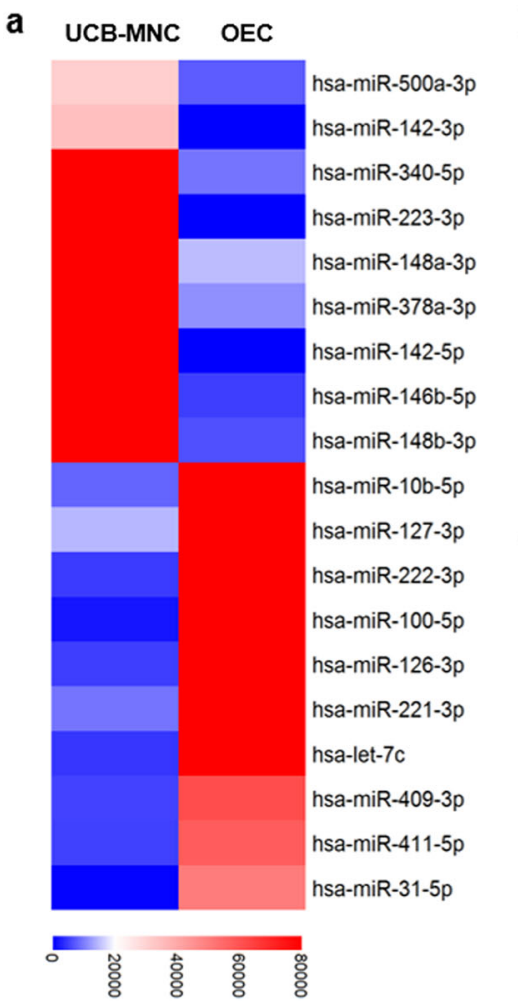

\section{b}

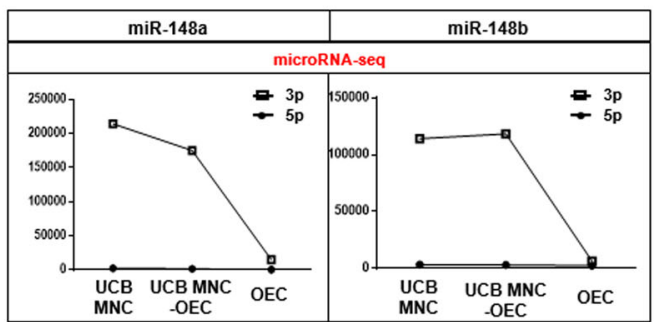

C

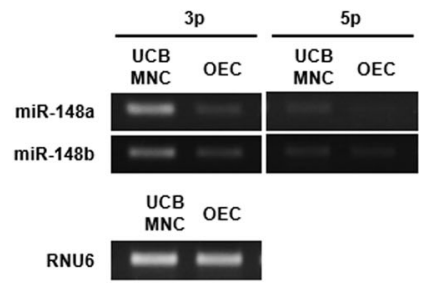

Fig. 1 Differentially expressed microRNAs were identified in UCB-MNCs and OECs. a A heat map of RNA sequencing data illustrates microRNAs (miRs) that are differentially expressed in UCB-MNCs and OECs. Red and blue indicate high and low-miR expression, respectively. Circulating MNCS were isolated from healthy human umbilical cord blood, and UCB-MNCs were cultured in EBM-2 to induce OEC differentiation. OEC identification and culture purity (90-95\%) were determined by the uptake of Dil-conjugated acLDL and by staining with EC-specific markers, including VE-cadherin. b Lower levels of miR-148a and miR-148b were observed in OECs than what was observed in UCB-MNCs. c The expression of miR-148a/b-3p and miR-148a/b-5p in UCB-MNCs and OECs was confirmed by RT-PCR. RNU6 mRNA was used as an internal control

DIANA Tools, Targetscan, and miRDB. There were 145 target genes for miR-148a-3p and 147 for miR-148b-3p that were predicted by all three algorithms (Fig. 2a). Of these, NRP1, ITGa5 (Integrin $\alpha$ ), and ROBO1 (Roundabout Guidance Receptor 1) contribute to EC function. To validate the putative miR-148a/b-3p targets, we next investigated the mRNA levels of NRP1, ITGa5, and ROBO1 in UCB-MNCs and OECs. The expression of NRP1, ITGa5, and ROBO1 was significantly enhanced in OECs and was inversely correlated with $\mathrm{miR}-148 \mathrm{a} / \mathrm{b}-3 \mathrm{p}$ expression (Fig. 2b). In addition, the mRNA and protein levels of NRP1, ITGa5, and ROBO1 were repressed by the overexpression of a miR-148a/b-3p mimic in ECs (Fig. 2c, d). Consistent with these data, RT-PCR data revealed that the transfection of a miR-148a/b-3p mimic in ECs reduced the mRNA and protein levels of the target genes in a time-dependent manner (Fig. 2e). Among the identified targets, we focused on NRP1, which is a nontyrosine kinase receptor for VEGF.

To investigate whether NRP1 was a direct target of miR$148 \mathrm{a} / \mathrm{b}-3 \mathrm{p}$, we constructed luciferase reporters containing the 3'UTR of NRP1 mRNA and either the wild-type or mutant seed sequence for miR-148a/b-3p. The luciferase reporters were cotransfected with a miR-control or miR148a/b-3p mimic into ECs. The reporter assay also indicated that miR-148a/b-3p inhibited NRP1 expression by directly binding to the $3^{\prime} \mathrm{UTR}$ of NRP1 mRNA (Fig. 2f).

\section{Upregulation of miR-148a/b-3p delays cell migration and inhibits the formation of lamellipodia in ECs}

Endogenous expression of miR-148a/b-3p in fully differentiated ECs is low; therefore, we hypothesized that increasing the miR levels in ECs would have a biological effect. We transfected ECs with miR-148-a/b-3p mimics and a miR-control to investigate the effect of miR-148a/b$3 \mathrm{p}$ overexpression on angiogenic properties (Supplementary Fig. S1). Because EC migration is an essential step of angiogenesis, we first performed a Transwell migration assay (Fig. 3a). Compared with the control cells, the migration of ECs treated with either miR-148a or miR-148b was reduced by greater than $40 \%$. VEGF stimulation enhanced cell motility, but the migration of ECs treated with the miR-148a/b mimics was less pronounced (Fig. 3b). VEGF-induced EC migration requires 


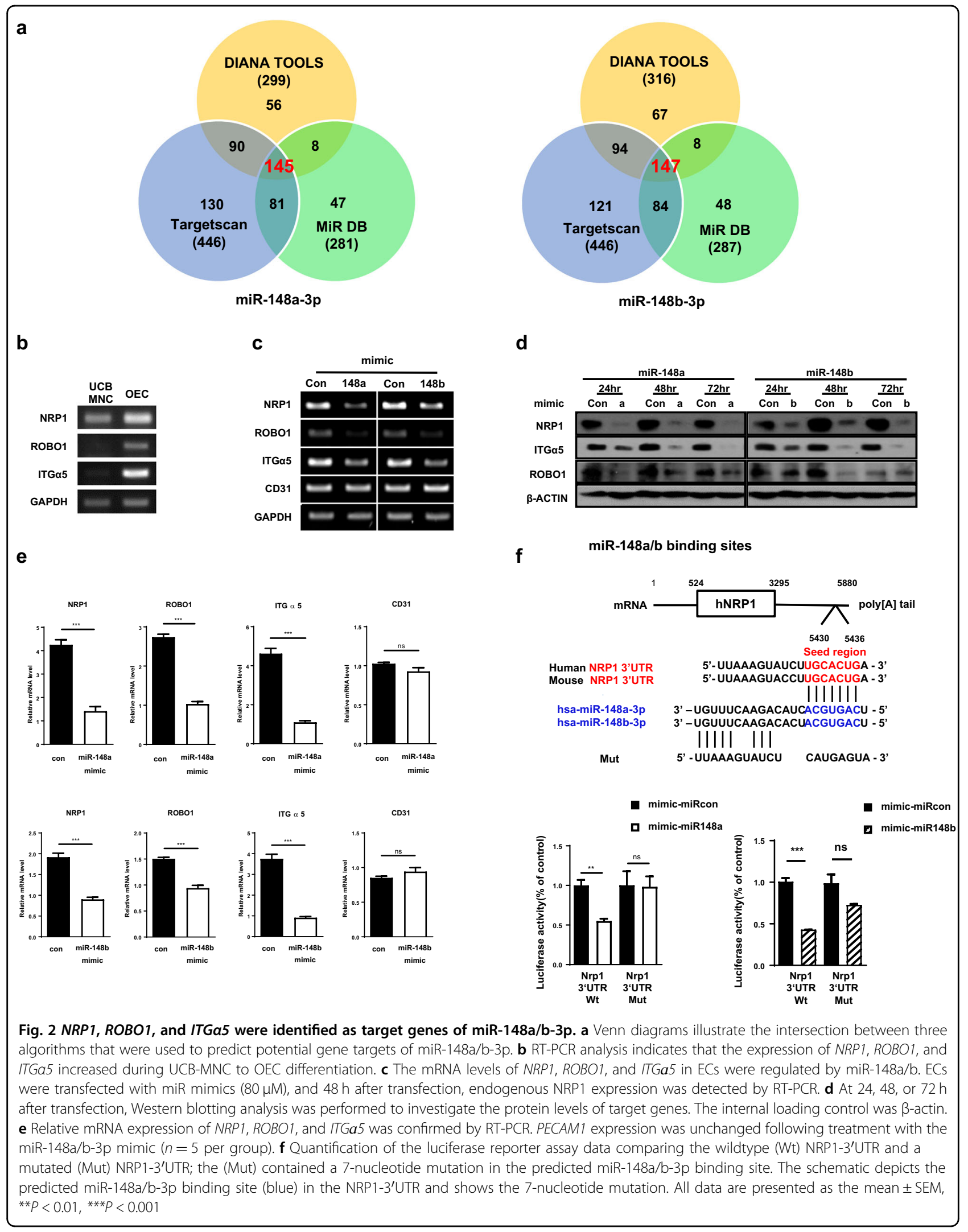




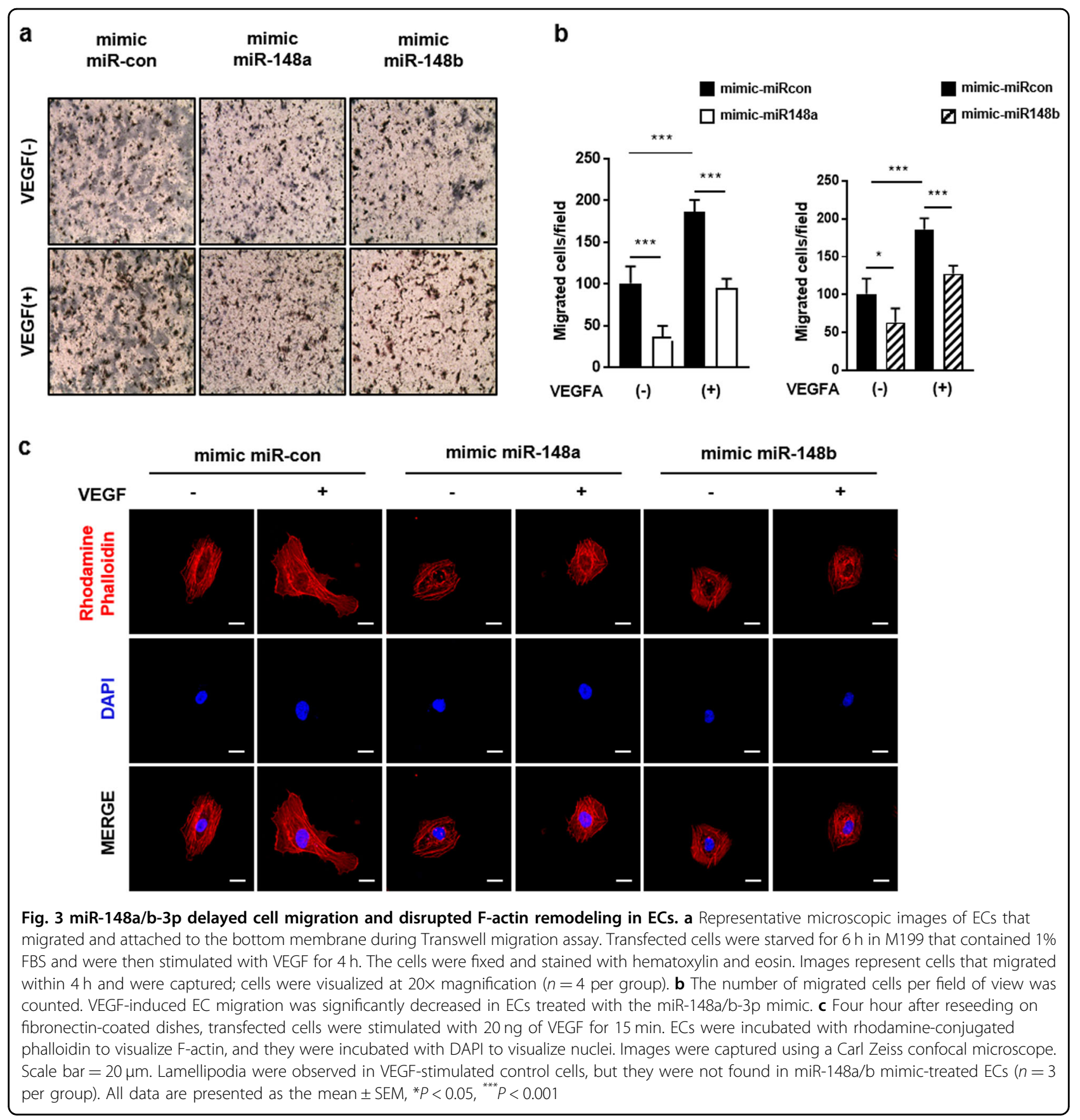

remodeling of the actin cytoskeleton into lamellipodia, which prompted us to investigate the effects of miR-148a/ b on EC morphology. Phalloidin was used to visualize filamentous actin (F-actin). In control cells, F-actin staining showed lamellipodia with branched actin networks, and tight parallel F-actin bundles were well organized. In addition, after VEGF stimulation, the ends of the actin filaments extended rapidly towards the leading edge (Fig. 3c). These characteristics are typically found in motile cells ${ }^{16}$. On the other hand, the miR-148a/b-3p mimic-treated ECs exhibited a contracted, round morphology, greater cortical actin structures, and fewer Factin stress fibers. In addition, VEGF stimulation did not induce cellular protrusions toward the leading edge in these mimic-treated cells. We found that miR-148a/b-3p mimic-treated ECs, unlike control cells, failed to migrate through gelatin-coated Transwells, and the cytoskeleton did not remodel into lamellipodia, even with VEGF stimulation. These results suggest that miR-148a/b-3p is an essential mediator of EC migration. 


\section{Overexpression of $\mathrm{miR} 148 \mathrm{a} / \mathrm{b}-3 \mathrm{p}$ inhibits angiogenic sprouting in vitro}

Because miR148-a/b-3p inhibited EC migration, we further investigated whether miR148-a/b-3p repressed angiogenesis using a fibrin gel sprouting assay that mimics neovascularization in vitro. miR-148a/b-3p impaired new sprout formation in fibrin gels (Fig. 4a). In the absence of VEGF, ECs transfected with miR148a/b-3p mimics formed fewer sprouts per bead and had a shorter cumulative sprout length when compared to control cells. VEGF stimulation increased the number of sprouts and the cumulative sprout length in both miR-148a/b-3p- and miR-control-transfected ECs (Fig. 4b-e). However, we noted that even with VEGF stimulation, the miR-148a/b3p mimic-treated ECs had a significantly lower mean number of sprouts and a shorter cumulative sprout length than the control cells did under identical stimulation conditions. These results indicated that miR-148a/b-3p may inhibit the formation of new vasculature.

\section{VEGFR2 signaling is regulated by miR-148a/b-3p}

We next investigated the effects of miR148a/b-3pmediated NRP1 inhibition on VEGFR2 signaling by stimulating ECs that overexpressed miR-148a/b-3p with VEGF. Although total VEGFR2 protein levels were increased, VEGFR2 and ERK phosphorylation induced by VEGF was decreased (Fig. 5a, b). These data suggest that miR-148a/b-3p exerts antiangiogenic effects by directly targeting NRP1 on the surface of ECs, which disrupts VEGFR2-mediated signaling. Consistent with previous reports, VEGFR2 alone does not fully activate VEGFR2 signaling without its coreceptor, NRP1. Decreased VEGFR2 phosphorylation contributed to the reduced activity of various downstream signaling substrates that are paramount to EC migration and sprouting.

Phosphorylation of other downstream signaling targets of VEGFR2, FAK (focal adhesion kinase) and ERK, was also decreased in cells overexpressing the miR-148a/b-3p mimics (Fig. 5a, b). We observed that the miR-148a/b-3p mimic disrupted lamellipodia formation in ECs (Fig. 3c). Other groups report that in the absence of p-FAK, cells at the edge of a wound do not form protruding lamellipodia. Coupled with our data, these findings suggest that the inhibition of NRP1 expression by miR-148a/b-3p in ECs reduces signaling downstream of VEGFR2 and results in suppressed migration, F-actin remodeling, and sprouting.

\section{Discussion}

ECs are critical to all aspects of vascular homeostasis, and EC dysfunction is directly associated with the development of vascular leakage, infectious diseases, stroke, and cancer angiogenesis ${ }^{17}$. The aim of this study was to investigate the role of miR-148a/b-3p in ECs. miR-148a/ b-3p belongs to the miR-148/152 family, which comprises
miR-148a, miR-148b, and miR- $152^{18}$. miR-148a and miR$148 \mathrm{~b}$, which are 22 nucleotides in length, are conserved in various species, including mice, dogs, and humans (Supplementary Fig. S2). The mature sequence of miR-148a/b has eight nearly identical seed sequences that differ by only two nucleotides. The structural similarity between miR-148a and miR-148b implies that the two miRs may perform similar biological functions. Using RNA-seq analysis, we found that the expression of $\mathrm{miR}-148 \mathrm{a} / \mathrm{b}-3 \mathrm{p}$ significantly decreased during differentiation of UCBMNCs into OECs. In addition, hsa-miR-142-3p, hsa-miR223-3p, hsa-miR-146b-5p, hsa-miR-10, hsa-miR-222, and miR-31-5p also showed expression differences that were similar to those observed for miR-148a/b-3p (Fig. 1a). Interestingly, these miRs are known to have angiogenic effects due to their regulation of EC function. For example, miR-142-3p promotes EC proliferation through regulation of Bcl-2-associated transcription factor 1 under hypertensive conditions ${ }^{19}$. miR-223-3p inhibits angiogenesis by suppressing the migration and proliferation of ischemic cardiac microvascular ECs in ischemic heart disease $^{20}$. In addition, upregulation of miR-146b-5p inhibits Ang-1 and causes LPS-induced inflammation in $\mathrm{ECs}^{21}$. These prior studies provided foundational evidence that miR-148a/b-3p could have important implications on $\mathrm{EC}$ function in angiogenesis. Using miR target prediction programs, we identified 132 common targets for miR$148 \mathrm{a} / \mathrm{b}$ in both humans and mice. The majority of the gene targets were overlapping and conserved between miR-148a and miR-148b, and they included NRP1, ITG $\alpha 5$, ROBO1, and DNMT1 (Supplementary Tables 1 and 2). Among these targets, we chose to examine NRP1 regulation by miR148a/b-3p. The luciferase assay results demonstrated that miR148a/b-3p directly targets the $3^{\prime}$ UTR of NRP1. Moreover, we found that the mRNA expression of NRP1 is specifically increased in OECs when compared to what is observed in UCB-MNCs. The expression of miR148a/b-3p was inversely correlated with NRP1 expression. Based on these data, we hypothesized that decreased miR-148a/b expression in OECs would upregulate NRP1 and alter EC function. Thus, we investigated how EC function was changed by increasing miR$148 \mathrm{a} / \mathrm{b}$ levels. Interestingly, miR148a/b-3p expression caused a decrease in NRP1 expression and led to antiangiogenic phenotypes. We confirmed that the overexpression of miR148a/b-3p inhibited migration and Factin remodeling in ECs. These findings are consistent with the cell morphology changes that occur when small inhibiting NRP1 RNA (siNRP1) is transfected into human dermal microvascular ECs (HDMECs). In HDMECs, siNRP1 inhibited fibronectin-induced Cdc42 activation and consequently impaired actin remodeling ${ }^{22}$. Furthermore, NRP1 is known to promote EC migration, which is stimulated by both VEGF and extracellular matrix cues $^{23}$. 
a

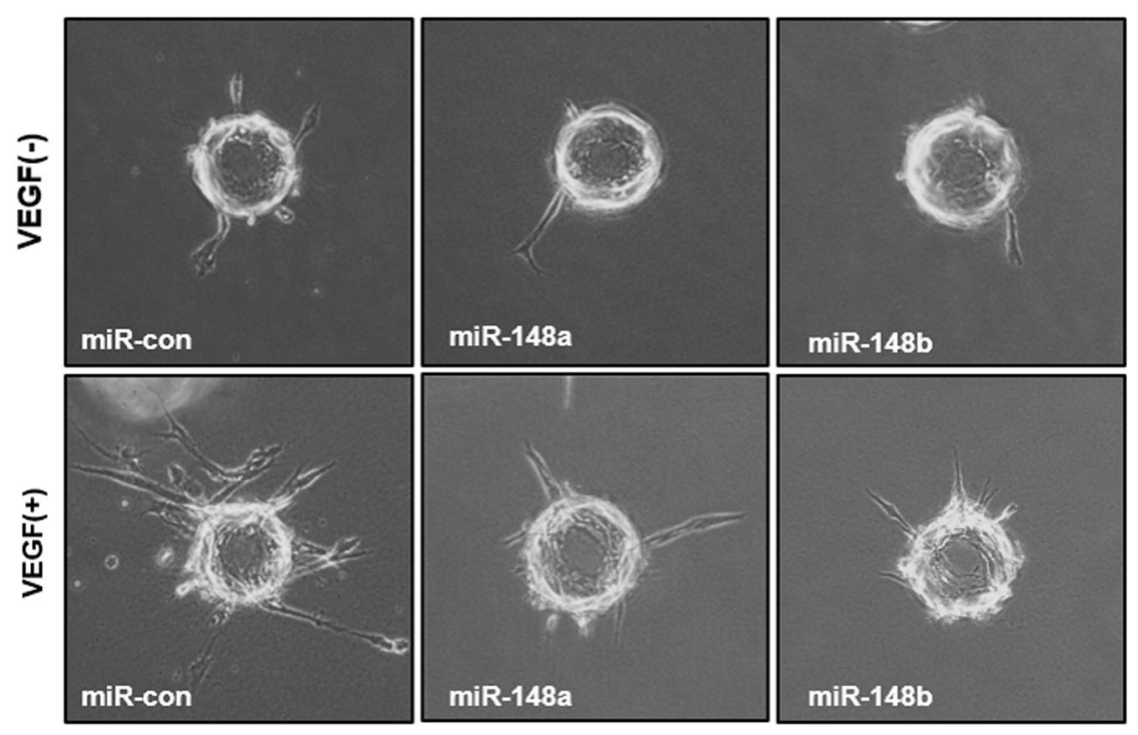

b

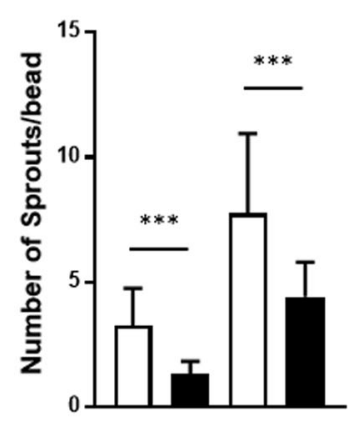

VEGFA
(-)
$(+)$

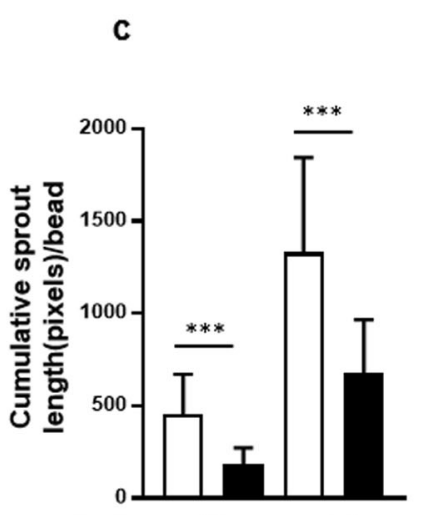

VEGFA
(-)
(+)
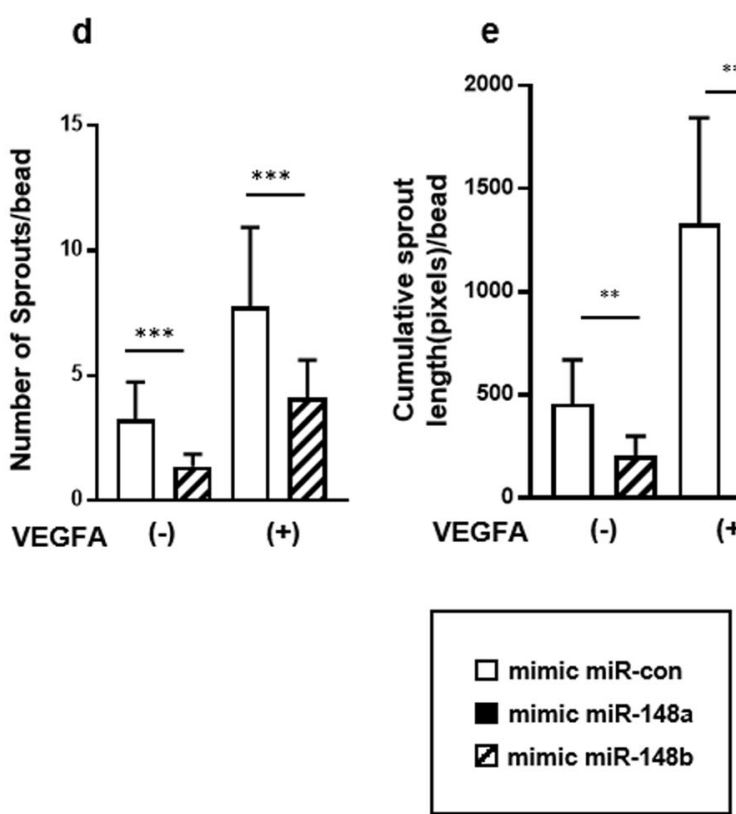

Fig. 4 miR-148a/b-3p impaired angiogenic sprouting in fibrin gels. a A sprouting assay was performed on HUVECs that were transfected with miR-control or miR-148a/b-3p mimics with or without VEGF $(30 \mathrm{ng} / \mathrm{mL})$ stimulation in $2 \%$ EBM media. Representative microscopic images show EC morphogenesis during in vitro angiogenesis in fibrin gels. Sprouts were observed on day 2 and continued to proliferate, migrate, branch, and form lumens on days 3-5 in culture. b Angiogenic sprouting was quantitatively analyzed by measuring the number of sprouts per bead and the cumulative sprout length per bead ( $n=10$ per group). All data are presented as the mean \pm SEM, ${ }^{* *} P<0.01,{ }^{* * *} P<0.001$

Although it was not a part of the present study, DNMT1 (DNA [cytosine-5-]methyltransferase 1), was also predicted by our data to be a potential target of miR-148a/b. DNA sequences that encode miRs can undergo abnormal DNA methylation, which influences their expression. In many types of cancers, miR-148a/b expression is low due to the hypermethylation of a CpG island in the miR-148a/b coding region ${ }^{10,24,25}$. In pancreatic cancer, overexpression of miR-148b lowers DNMT1 expression and contributes to the modified methylation status of tumor suppressor genes $^{25}$. In breast cancer, increased DNMT1 expression lowers DNMT1 expression and contributes to enhanced hyperactivation of the PI3K/AKT and ERK pathways that promote tumor growth ${ }^{18}$. These data support the premise 


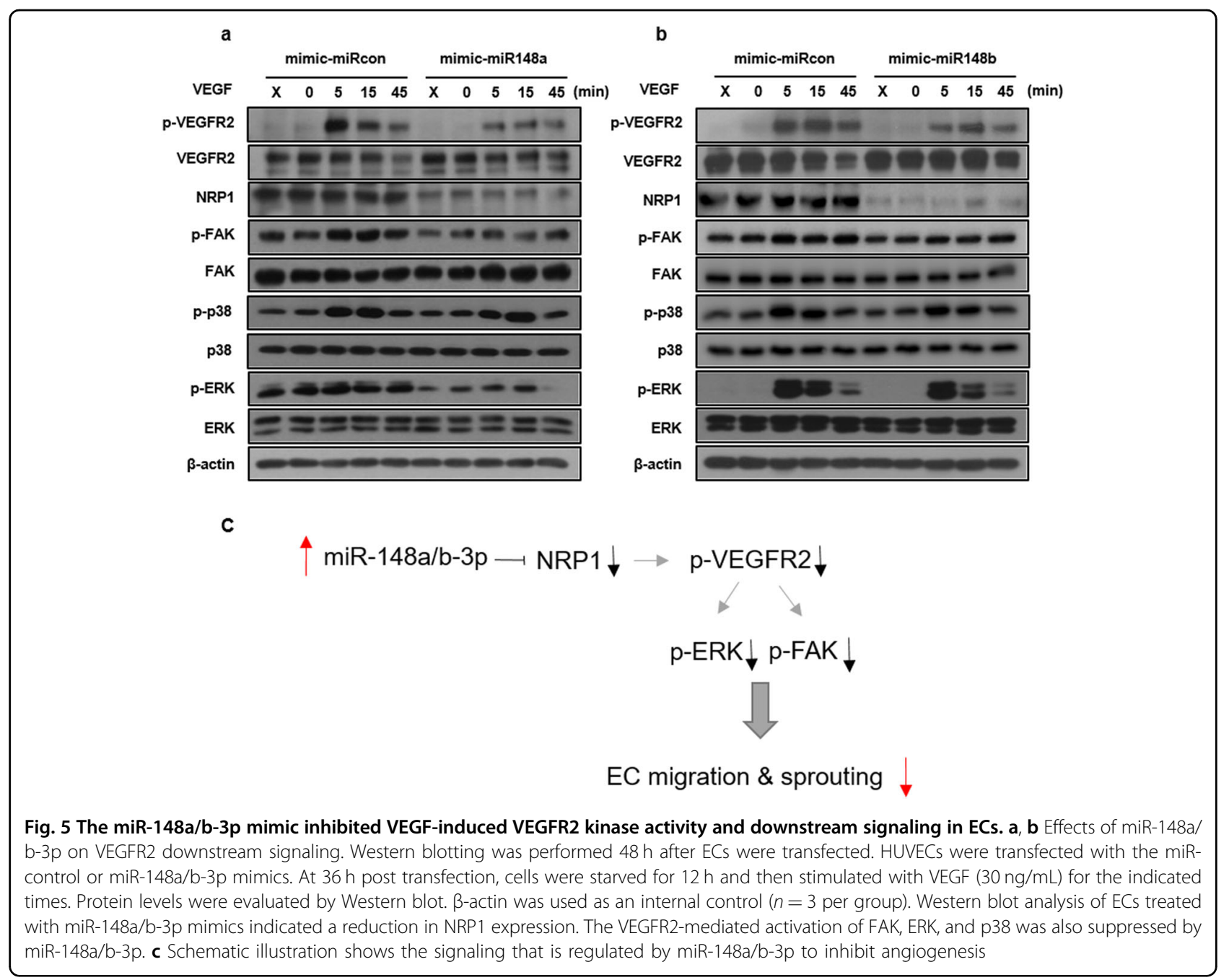

that abnormal expression of miR-148a/b, which regulates DNMT1, promotes tumorigenesis.

Our study revealed that miR-148a/b-3p plays an important role not only in tumorigenesis but also in normal physiological EC function. Indeed, miR148a and miR148b are major regulators of EC migration and sprouting in response to VEGF. As summarized in Fig. 5c, upregulating the expression of $\mathrm{miR}-148 \mathrm{a} / \mathrm{b}-3 \mathrm{p}$ in ECs from its typically low endogenous levels severely inhibited VEGF-induced activation of VEGFR2 and subsequent downstream signaling by directly targeting NRP1. The inhibition of VEGFR2 signaling inhibited migration, Factin remodeling, and sprouting in ECs. We further confirmed that miR-148a/b-3p markedly reduced EC proliferation through the EGFR signaling pathway, which is also known to regulate NRP1 (Supplementary Fig. S3) ${ }^{26,27}$. Recently, a human IgG1 monoclonal antibody was developed that blocks the NRP1 b1b2 domain, which is in the VEGF binding site, and the antibody was used to treat vascular ECs. Notably, the antibody blocked angiogenesis and vascular remodeling ${ }^{28}$. Collectively, these data suggest that miR148a/b-3p may be regulating antiangiogenic miRs in vascular disease and VEGF-driven angiogenesis by inhibiting NRP1.

\section{Acknowledgements}

This research was supported by the Basic Science Research Program through the National Research Foundation of Korea (NRF), and it was funded by the Ministry of Education, Science and Technology (MEST; Grant 2019R1A2C3007142), and the Bio \& Medical Technology Development Program of the NRF, funded by the Korean government, MSIP (NRF2015M3A9B6066835). This work was also supported by a grant from the Korea Health Technology R\&D Project through the Korea Health Industry Development Institute (KHIDI), and it was funded by the Ministry of Health \& Welfare, Republic of Korea (Grant number HI16C1501).

\section{Author details}

'Department of Biochemistry, College of Life Science and Biotechnology, Yonsei University, Seoul 03722, Korea. ${ }^{2}$ Vascular System Research Center, Kangwon National University, Chuncheon, Kangwon 24341, Republic of Korea

Conflict of interest

The authors declare that they have no conflict of interest. 


\section{Publisher's note}

Springer Nature remains neutral with regard to jurisdictional claims in published maps and institutional affiliations.

Supplementary information accompanies this paper at https://doi.org/ 10.1038/s12276-019-0344-x.

Received: 13 May 2019 Revised: 20 September 2019 Accepted: 26 September 2019.

Published online: 13 November 2019

\section{References}

1. Felmeden, D. Angiogenesis: basic pathophysiology and implications for disease. Eur. Heart J. 24, 586-603 (2003).

2. Ferrara, N., Gerber, H. P. \& LeCouter, J. The biology of VEGF and its receptors. Nat. Med. 9, 669-676 (2003).

3. Abhinand, C. S., Raju, R., Soumya, S. J., Arya, P. S. \& Sudhakaran, P. R. VEGF-A VEGFR2 signaling network in endothelial cells relevant to angiogenesis. J. Cell Commun. Signal. 10, 347-354 (2016).

4. Prahst, C. et al. Neuropilin-1-VEGFR-2 complexing requires the PDZ-binding domain of neuropilin-1. J. Biol. Chem. 283, 25110-25114 (2008).

5. Ballmer-Hofer, K., Andersson, A. E., Ratcliffe, L. E. \& Berger, P. Neuropilin-1 promotes VEGFR-2 trafficking through Rab11 vesicles thereby specifying signal output. Blood 118, 816-826 (2011).

6. Miao, H. Q., Lee, P., Lin, H., Soker, S. \& Klagsbrun, M. Neuropilin-1 expression by tumor cells promotes tumor angiogenesis and progression. FASEB J. 14, 2532-2539 (2000).

7. Wu, Y. Y. et al. miR-320 regulates tumor angiogenesis driven by vascular endothelial cells in oral cancer by silencing neuropilin 1. Angiogenesis 17, 247-260 (2014).

8. Gebert, L. F. R. \& MacRae, I. J. Regulation of microRNA function in animals. Nat. Rev. Mol. Cell Biol. 20, 21-37 (2019).

9. Pan, L. et al. Decreased expression and clinical significance of miR-148a in hepatocellular carcinoma tissues. Eur. J. Med. Res. 19, 68 (2014).

10. Zhu, A. et al. MicroRNA-148a is silenced by hypermethylation and interacts with DNA methyltransferase 1 in gastric cancer. Med. Oncol. 29, 2701-2709 (2012).

11. Wang, W. et al. miR-148a-3p suppresses epithelial ovarian cancer progression primarily by targeting c-Met. Oncol. Lett. 15, 6131-6136 (2018).

12. Wang, Y. et al. miR-148b-3p functions as a tumor suppressor in GISTs by directly targeting KIT. Cell Commun. Signal 16, 16 (2018).

13. Maeng, Y. S. et al. Endothelial progenitor cell homing: prominent role of the IGF2-IGF2R-PLCbeta2 axis. Blood 113, 233-243 (2009).
14. Kimura, T. et al. CXCL8 enhances the angiogenic activity of umbilical cord blood-derived outgrowth endothelial cells in vitro. Cell Biol. Int. 35, 201-208 (2011).

15. Nakatsu, M. N., Davis, J. \& Hughes, C. C. A previously established fibrin gel bead assay was optimized to study angiogenesis. J. Vis. Exp. 186 (2007).

16. Lamalice, L., Le Boeuf, F. \& Huot, J. Endothelial cell migration during angiogenesis. Circ. Res. 100, 782-794 (2007).

17. Rajendran, P. et al. The vascular endothelium and human diseases. Int J. Biol. Sci. 9, 1057-1069 (2013).

18. Xu, Q. et al. A regulatory circuit of miR-148a/152 and DNMT1 in modulating cell transformation and tumor angiogenesis through IGF-IR and IRS1. J. Mol. Cell Biol. 5, 3-13 (2013).

19. Bao, $\mathrm{H}$. et al. Platelet-derived microparticles promote endothelial cell proliferation in hypertension via miR-142-3p. FASEB J. 32, 3912-3923 (2018).

20. Dai, G. H. et al. MicroRNA-223-3p inhibits the angiogenesis of ischemic cardiac microvascular endothelial cells via affecting RPS6KB1/hif-1a signal pathway. PLOS ONE 9, e108468 (2014).

21. Echavarria, R. et al. Angiopoietin-1 inhibits toll-like receptor 4 signalling in cultured endothelial cells: role of miR-146b-5p. Cardiovasc. Res. 106, 465-477 (2015).

22. Fantin, A. et al. NRP1 regulates CDC42 activation to promote filopodia formation in endothelial tip cells. Cell Rep. 11, 1577-1590 (2015).

23. Raimondi, $C$. et al. Imatinib inhibits VEGF-independent angiogenesis by targeting neuropilin 1-dependent ABL1 activation in endothelial cells. J. Exp. Med. 211, 1167-1183 (2014).

24. Long, X. R., He, Y., Huang, C. \& Li, J. MicroRNA-148a is silenced by hypermethylation and interacts with DNA methyltransferase 1 in hepatocellular carcinogenesis. Int. J. Oncol. 44, 1915-1922 (2014).

25. Azizi, M. et al. MicroRNA-148b and microRNA-152 reactivate tumor suppressor genes through suppression of DNA methyltransferase-1 gene in pancreatic cancer cell lines. Cancer Biol. Ther. 15, 419-427 (2014).

26. Rizzolio, S. et al. Neuropilin-1-dependent regulation of EGF-receptor signaling Cancer Res. 72, 5801-5811 (2012).

27. Li, L. et al. Neuropilin-1 is associated with clinicopathology of gastric cancer and contributes to cell proliferation and migration as multifunctional coreceptors. J. Exp. Clin. Cancer Res. 35, 16 (2016).

28. Weekes, C. D. et al. A phase I study of the human monoclonal anti-NRP1 antibody MNRP1685A in patients with advanced solid tumors. Invest. New Drugs 32, 653-660 (2014). 